\title{
Hypertension in adolescents and young adults at a tertiary clinic in Cape Town, South Africa
}

\author{
Ilhaam Esack (ESCILH001@myuct.ac.za) \\ Brian Rayner (Brian.Rayner@uct.ac.za) \\ Phetho Mangena (phetho@gmail.com)
}

Supervisors: Professor Brian Rayner ORCID: orcid.org//0000-0002-0167-9591; Dr Phetho Mangena

Faculty of Health Sciences, University of Cape Town

\begin{abstract}
Background: The prevalence of essential hypertension in children and adolescents has increased dramatically over the past decade attributed to a rising prevalence of obesity, high salt diet and lack of exercise. Traditionally high blood pressure in adolescents required extensive work up to determine a cause, but given the changing demographics this policy needs to be reviewed. Objectives/method: The study aimed describe the risk factors, demographics, target organ damage and aetiology of hypertension in a cohort of young hypertensives (aged 15 - 30 years) referred to a tertiary hypertension clinic at Groote Schuur Hospital, by retrospectively reviewing the folders over a three-month period. Results: Thirty eight patients were identified with a mean age of 22 years. Essential hypertension was diagnosed in $82 \%$ of patients, and $71 \%$ of patients had a family history of hypertension. The median systolic blood pressure (BP) at first visit was $132 \mathrm{mmHg}$ and diastolic BP $84 \mathrm{mmHg}$. The median BMI was $25.4 \mathrm{~kg} / \mathrm{m}^{2}$ and $68 \%$ had some form of target organ damage. Twenty six percent were current smokers and $8 \%$ had abused metamphetamines. Recognised secondary causes of hypertension were renal artery stenosis (four) and primary aldosteronism (one). Conclusions: In a cohort of young hypertensives patients the dominant cause was essential hypertension. Although the prevalence of obesity was surprisingly low compared to previously published studies, significant lifestyle issues were identified. 68\% had target organ damage suggesting the disease was not benign. These preliminary results indicate that larger cohorts need to be studied to develop new policies for assessment and treatment of young people with hypertension in South Africa.
\end{abstract}

Keywords: hypertension; adolescents; aetiology; target organ damage; risk factors 


\section{Background}

A systematic analysis done in 2010 for the Global Burden of Disease report showed that hypertension is the "leading risk factor for global mortality" and was the cause of $7 \%$ of deaths worldwide in that year (Lim et al. 2012: 2224). Hypertension, also known as high BP, is defined as a persistent elevation of systolic BP $\geq 140 \mathrm{mmHg}$ and/or a diastolic BP $\geq 90 \mathrm{mmHg}$ in adults (Seedat et al. 2014: 288). In the majority there is no known underlying cause such as kidney disease, renal artery stenosis, coarctation of the aorta or endocrine causes and is termed essential hypertension. It has been traditionally taught that hypertension in young people is more likely to have a secondary cause and therefore required extensive investigation.

\section{Trends in BP among young people}

Adult BP is generally higher than that of children and the BP of children generally increases in the first 20 years of life (Alan 1996: 1968). Studies conducted in Israel and South Africa (Tirosh et al 2010: 203; Kagura et al 2015: 1; Israeli et al 2006: 708) have shown that despite the body mass index (BMI) of young females being higher than that of young males, the prevalence of pre-hypertension and hypertension is higher in males.

In South Africa there has been a dramatic rise in the prevalence of hypertension in young people. According to the 2008 National Income Dynamics Study in South Africa (Bradshaw et al 2011:3), 11\% of males aged 15-24 (compared to 4\% in the 1998 SADHS) and $20 \%$ of males aged $25-34$ (compared to $10 \%$ in 1998) have hypertension. In females, $12 \%$ of $15-24$ year olds (compared to $7 \%$ in 1998 ) and $24 \%$ of $25-34$ year olds (compared to $15 \%$ in 1998) have hypertension. This has been mirrored in other parts of the world (Dong et al 2013: 997; Assadi 2012: 1013). In 2014 the global prevalence of hypertension in individuals older than 18 years was 22\% (WHO 2014:67-68). This high prevalence calls for increased attention to the prevention and management of this disease. 


\section{Factors affecting BP in young people}

There have been numerous studies demonstrating that the risk factors for hypertension in adolescents are obesity, low birth weight, family history of hypertension and diabetes, and sedentary behavior.

From 2002 and 2008 the South African National Youth Risk Behavior Survey (Reddy et al 2012: 265) showed that the rate of obesity increased in adolescents (grade $8-11$ ) from $1.6 \%$ to $3.3 \%$ in males and $5.0 \%$ to $7.5 \%$ in females respectively. Ippisch \& Daniels (2008: 179) stated that childhood and adolescent obesity is one of the strongest predictors of adult hypertension. In a study showing trends of high BP in US children and adolescents, $\mathrm{Xi}$ et al (2015: 1) demonstrated that programs that prevent obesity at earlier ages can decrease BP in later years.

There is also a familial influence on high BP with studies showing an increased prevalence in children with a family history of hypertension when compared to children from normotensive families (Alan 1996: 1968) suggesting an underlying genetic predisposition.

\section{Diagnosis and Treatment}

Contemporary guidelines such as the $4^{\text {th }}$ report of National Health and Nutrition Examination Survey (NHANES) define pre-hypertension and hypertension based on percentile reference charts for children and adolescents, which take into account age, gender and height (Falkner \& Daniels 2004: 387). Other factors include waist-height ratio, waist-BMI ratio and BP-height ratio. In young children especially, the diagnostic criteria for hypertension can be complicated (for example, the percentile charts can be time consuming to interpret compared to the diagnosis of hypertension in adults, for which there is a single normal range) resulting in a large number of undiagnosed cases (Lu et al. 2011: 162).

Staging of hypertension is determined according to percentile charts as well as from evidence of any end-organ damage such as retinopathy, left ventricular hypertrophy (LVH) and nephropathy (Falkner \& Daniels 2004: 387). This is important in distinguishing between those patients who require antihypertensive medication and those for whom initial conservative treatment would be sufficient. 
The currently recommended assessment for a young patient with hypertension ('full workup') includes an echocardiogram, 24 hour ambulatory BP monitoring, renal function tests, electrolyte tests, endocrine tests, CT angiography and renal ultrasound (Ippisch \& Daniels 2008:180). These tests are done in order to exclude secondary causes for raised blood pressure. However, due to the rising prevalence of essential hypertension in adolescents, it is questionable as to whether this is necessary and more research needs to be done in order to determine the feasibility thereof.

The South African Hypertension Guidelines advocate for lifestyle changes in all hypertensive patients. This includes weight loss, exercise, reduced alcohol use, increased potassium intake and reduced salt intake. In the case that lifestyle changes have failed, or where there is target-organ damage, anti-hypertensive drugs should be used. This includes: beta-blockers, ACE inhibitors, diuretics, calcium channel blockers and alpha-blockers. The lowest dose of a single drug should be administered first and slowly increased until the desired BP is reached. In the case of severe side effects or unchanged BP, a drug from a different class should be added or substituted (Rayner 2010: 272).It is important for intervention to be started timeously in young hypertensives in order to reduce the risk of developing hypertension in adulthood (Tu et al. 2011: 818).

\section{Methods}

\section{Study design}

In this retrospective, non-analytic (I AM NOT SURE THE STUDY CAN BE DESCRIBED AS "NON-ANALYTIC" - CONSIDER DELETING THIS) descriptive cohort study, folders of patients between the ages of 15 and 30 who visited the Groote Schuur Hospital Hypertension Clinic from 6 January 2014 to 3 March 2014 were reviewed. The data collected included clinical and laboratory data, investigations, causes of hypertension, and target organ damage. 


\section{Measures}

Clinical data:

Demographic data: age at first visit, smoking status, drug use, duration of hypertension, family history of diabetes and hypertension, presence of diabetes mellitus, number of anti-hypertensives used, and hypertension diagnosis (essential hypertension or a secondary cause). Anthropomorphic data: height, weight and waist circumference were measured by the nurses in the clinic prior to the patient's appointment date in accordance with routine clinic procedures. BMI was calculated: weight $(\mathrm{kg}) /$ height $^{2}\left(\mathrm{~m}^{2}\right)$. BMI categories used were: underweight: under 20; ideal weight: 20-24; overweight: 25-30; obese: 30-40; morbidly obese: $40+$.

\section{Target Organ Damage:}

- All patients receive an ECG prior to their appointment and presence of left ventricular hypertrophy (LVH) was determined using Sokolow-Lyon criteria and/or Cornell voltage criteria.

- Fundoscopy is routinely performed to diagnose retinopathy and the grade was also recorded (grade 1: silver wiring, grade 2: AV nipping, grade 3: haemorrhages, grade 4: papilloedema).

- Nephropathy was diagnosed using the urine albumin/creatinine ratio and estimated GFR $<60 \mathrm{mls} / \mathrm{min}$. Microalbuminuria was defined as albumin/creatinine ratio of $3-30 \mathrm{mg} / \mathrm{mmol}$ and macroalbuminuria is defined as albumin/creatinine ratio of more than $30 \mathrm{mg} / \mathrm{mmol}$.

- Presence of ischaemic heart disease or coronary artery disease, previous cerebrovascular incidents and presence of peripheral vascular disease was also recorded.

Blood Pressure: automated readings in a seated position are done for each patient at each visit to the clinic. The average of the last 3 recorded BPs was used in order to exclude white coat hypertension from the first readings. 
Laboratory data:

Serum potassium, creatinine (derived from this was the MDRD estimated Glomerular Filtration Rate (eGFR)), fasting glucose and lipogram, plasma renin concentration, plasma aldosterone concentration, renin-aldosterone ratio, urine albumin-creatinine ratio and $\mathrm{R} 536 \mathrm{Q}$ gene mutation of the epithelial sodium channel. The R563Q mutation is associated with hypertension with decreased activity of renin and aldosterone in black Africans and those with mixed ancestry in South Africa (Jones et al. 2012: 1286). Further special investigations such as abdominal/renal ultrasound and renal CT angiogram were only done at the discretion of the consultant in the clinic when there is a suspicion of an underlying secondary cause.

\section{Data management}

The data collected was entered onto, and analyzed using a Microsoft Excel spreadsheet.

\section{Ethical considerations}

The University of Cape Town, Faculty of Health Sciences Human Research Ethics Committee approved this project in March 2015.

\section{Results}

\section{Demographic results}

Thirty eight patients were entered into the study with a mean age of 22 years. Twenty nine percent of the patients were aged $15-19$, $42 \%$ were $20-25$ and $29 \%$ were 26-30. The median systolic BP at first visit was $132 \mathrm{mmHg}$ and diastolic BP at first visit was $84 \mathrm{mmHg}$.

Essential hypertension was diagnosed in $82 \%$ of patients and $18 \%$ had a secondary cause for hypertension. Of the group with secondary hypertension one patient had primary hyperaldosteronism, four had renal artery stenosis (three of these with Takayasu arteritis) and in one patient there was an unspecified secondary cause. 
One patient in the sample was a current abuser of metamphetamine whilst two patients reported previous use. Twenty six percent were smokers, and $11 \%$ were exsmokers.

Thirty nine percent of patients in the cohort had duration of hypertension of less than one year, $42 \%$ had it for $1-3$ years, $11 \%$ had it for more than 3 years and for $8 \%$ there was no data available.

Nearly three quarters $(71 \%)$ of patients had a family history of hypertension, $26 \%$ of patients had a family history of diabetes mellitus and $18 \%$ had a family history of both conditions. One patient in the cohort had diabetes mellitus.

In terms of target organ damage (TOD) $68 \%$ of the patients had evidence for hypertensive damage with a high prevalence of LVH, CKD and retinopathy (Table 1).

\section{Anthropomorphic data}

The median BMI for the cohort was $25.4 \mathrm{~kg} / \mathrm{m}^{2}$. In terms of the distribution of BMI in the cohort: $42 \%$ had a normal BMI, $18 \%$ were overweight, $24 \%$ were obese, $3 \%$ were morbidly obese and $13 \%$ did not have sufficient data available in order to derive the BMI. 


\section{Target Organ Damage (TOD)}

Table 1: Target Organ Damage in the cohort

\begin{tabular}{|l|l|l|}
\hline \multicolumn{2}{|l|}{ Target Organ Damage (TOD) } \\
\hline & n \\
\hline Any TOD & 26 & $\%$ \\
\hline LVH & 23 & 68 \\
\hline Retinopathy & 9 & 61 \\
\hline grade 1 & 6 & 35 \\
\hline grade 2 & 3 & 23 \\
\hline & \multicolumn{2}{|l|}{} \\
\hline Nephropathy & 1 & 12 \\
\hline eGFR <60 ml/min/1,73m² & 1 & 4 \\
\hline micro albuminuria & 3 & 12 \\
\hline macro albuminuria & 6 & 23 \\
\hline \multicolumn{2}{|l|}{} \\
\hline Coronary artery disease & 1 & 4 \\
\hline
\end{tabular}

\section{Laboratory data and special investigations}

Selected laboratory data is shown in Table 2. Overall $16 \%$ of the cohort had CKD as evidenced by eGFR $<60 \mathrm{ml} / \mathrm{min}$ and microalbuminuria or macroalbumnuria. Metabolic syndrome was present in 3 patients and 2 had a fasting glucose in the diabetic range.

One patient in the cohort had primary aldosteronism (in the form of an adrenal tumour). This patient had a plasma renin concentration of $5 \mathrm{mlU} / \mathrm{l}$, a plasma aldosterone level of $668 \mathrm{pmol} / \mathrm{l}$ and an Aldosterone/Renin ratio of 133.6. Three patients had the $\mathrm{R} 563 \mathrm{Q}$ mutation of the $\mathrm{ENaC}$ gene.

Abdominal U/S was done in 8 patients and all tests were normal. CT scan was performed in 10 patients and demonstrated renal artery stenosis in 4 patients (three with Takayasu arteritis) and an adrenal tumour in one. 
Table 2: Laboratory data for different parameters

\begin{tabular}{|c|c|c|}
\hline Parameter & frequency & \begin{tabular}{|l|l}
$\%$ & of \\
cohort
\end{tabular} \\
\hline \multicolumn{3}{|l|}{ Potassium (mmol/l) } \\
\hline$<3,5$ & 0 & 0 \\
\hline $3,5-5,5$ (normal) & 38 & 100 \\
\hline$>5,5$ & 0 & 0 \\
\hline \multicolumn{3}{|l|}{ Glucose (mmol/l) } \\
\hline$<4$ & 1 & 3 \\
\hline 4-7,8 (normal) & 34 & 89 \\
\hline$>7,8$ & 2 & 5 \\
\hline \multicolumn{3}{|l|}{ eGFR $\left(\mathrm{ml} / \mathrm{min} / 1,73 \mathrm{~m}^{2}\right)$} \\
\hline$<60$ & 1 & 3 \\
\hline$>60$ & 37 & 97 \\
\hline \multicolumn{3}{|l|}{ uric acid (mmol/l) } \\
\hline$<0,2$ & 1 & 3 \\
\hline $0,2-0,42$ (normal) & 33 & 87 \\
\hline$>0,42$ & 4 & 11 \\
\hline \multicolumn{3}{|l|}{ total cholesterol $(\mathrm{mmol} / \mathrm{l})$} \\
\hline$<5$ (normal) & 26 & 68 \\
\hline$>5$ & 11 & 29 \\
\hline no results & 1 & 3 \\
\hline \multicolumn{3}{|l|}{$\mathrm{HDL}(\mathrm{mmol} / \mathrm{l})$} \\
\hline$>1,2$ & 11 & 29 \\
\hline$<1,2$ & 11 & 29 \\
\hline no results & 16 & 42 \\
\hline \multicolumn{3}{|l|}{ alb/creat (mg/mmol) } \\
\hline$<3$ (normal) & 28 & 74 \\
\hline 3 to 30 (microalbuminuria) & 3 & 8 \\
\hline$>30$ (macroalbuminuria) & 6 & 16 \\
\hline no results & 1 & 3 \\
\hline \multicolumn{3}{|l|}{ R536Q gene mutation } \\
\hline positive & 3 & 8 \\
\hline negative & 27 & 71 \\
\hline no result & 9 & 24 \\
\hline
\end{tabular}




\section{Discussion}

The data have shown that the majority of the patients in this cohort were diagnosed with essential hypertension. This is in agreement with the large amount of epidemiological evidence, which shows that the prevalence of essential hypertension is on the rise. This suggests that even in this young age routine extensive work up for secondary hypertension is not warranted and indicates the need for a focused approach based on detailed history and examination.

Metamphetamine abuse in the Cape Town area is major epidemic. Jones and Rayner (2015:199) have shown in a study conducted in Cape Town, that the use of methamphetamines is associated with severe hypertension, mesangiocapillary glomerulonephritis and chronic kidney disease. The three patients who had a history of illicit drug use had all used amphetamines (commonly known in the country as "tik").

Although the majority of the cohort did not smoke, about $30 \%$ are either current or ex-smokers. Smoking is not a cause of hypertension but it is a major remedial cardiovascular risk factor. Counseling patients to quit smoking should be a priority when advising patients about lifestyle change.

Most patients had duration of hypertension of 1-3 years. The next largest proportion was for less than a year and the least amount of patients had duration of more than 3 years. This is concerning as hypertensive patients should have their BP controlled timeously in order to reduce the target organ damage incurred.

As seen in Table 1, 45\% of patients in the cohort were overweight, obese or morbidly obese and $48 \%$ of patients have normal BMI. This is surprising because it contests the current hypothesis that adolescent hypertension is strongly linked to obesity. Most (71\%) had a family history of hypertension suggesting an underlying genetic predisposition in addition to lifestyle issues.

As seen in table two, a large proportion of patients in this cohort had some form of target organ damage mainly in the form of LVH and nephropathy. This suggests that in this cohort hypertension is not following a benign course. TOD is a strong 
marker of future cardiovascular and renal events and its presence should prompt the institution of appropriate antihypertensive therapy in addition to lifestyle.

From Table 2, serum potassium levels were unremarkable. Although, most of the patients' fasting glucose levels were normal, but in $5 \%$ this was raised, which could suggest the presence of underlying diabetes mellitus.

Raised uric acid levels were present in $11 \%$ of patients. Most of the patients' uric acid levels were normal, as seen in table two. There is growing evidence showing a correlation between elevated serum uric acid and essential hypertension in adolescents (Feig \& Johnson 2007: 79). This suggests that in these patients, elevated serum uric acid levels could be a mediator of their hypertension.

Most patients had normal cholesterol levels but $11 \%$ had raised cholesterol, as seen in Table 8. This further emphasizes the need for lifestyle changes. In some patients, further medication was prescribed in order to normalize this in order to reduce the risk of atherosclerosis.

Remedial causes of hypertension were identified in 5 patients. The patient with Conn's syndrome can be cured with resection of the adrenal tumour and the patients with renal artery stenosis can undergo stenting or vascular reconstruction to cure/ameliorate their hypertension. CT scanning had a high pick up rate in those undergoing the procedure.

Testing for the mutation in the R536Q gene mutation showed $11.8 \%$ of patients were positive. These patients could be considered for treatment with amiloride, the specific inhibitor of the ENaC.

\section{Strengths and limitations}

A strong point of this study is that there are many variables, which can be used to fully describe the cohort. Laboratory tests are routinely done and were easily accessible. The ages of the patients were also fairly well distributed within the category being studied. 
The main limitation was the small sample size. Ideally, a study of this design should have at least double the number of patients.(I WOULD SUGGEST A LOT MORE - CONSIDER DELETING THE ENTIRE SENTENCE) Some of the notes were also incomplete, making analysis difficult and the results and conclusions potentially inaccurate. Another limitation was the use of ECG criteria to diagnose LVH, which is not validated in young patients less than 32 years of age. The echocardiogram is considered to be the 'gold standard' in diagnosing LVH and would be done in order to confirm the presence of LVH. Due to resource constraints echocardiography was not performed in the majority of patients.

\section{Conclusions and Recommendations}

In this retrospective survey we demonstrated that the majority of young hypertensives had essential hypertension suggesting that recommendations to routinely investigate for secondary causes should be revised. Lifestyle issues were important with high levels of smoking, overweight or obesity in $42 \%$, and $7.8 \%$ either currently or historically abusing amphetamines. There were high levels of target organ damage in the form of nephropathy and LVH suggesting that many patients need drug treatment in addition to lifestyle changes. Over $50 \%$ of the cohort were not obese suggesting that in the South African population other factors are operative in pathogenesis of essential hypertension such as the R563Q mutation.

In conclusion this initial cohort study has demonstrated important findings but this needs to be confirmed by the inclusion of greater numbers into this ongoing cohort study. 


\section{References}

Alan, R. 1996. Hypertension in Children. New England Journal of Medicine. 335(26): 1968-1973.

Assadi, F. 2012. The growing epidemic of hypertension among children and adolescents: A challenging road ahead. Pediatric Cardiology. 33:1013-1020.

Bradshaw, D; Steyn, K; Levitt, N and Nojilana, B. Non-Communicable Diseases - A race against time. 2011.

Dong, B; Wang, H; Wang, Z; Liu, J and Ma, J. 2013. Trends in BP and Body Mass Index Among Chinese Children and Adolescents from 2005 to 2010. American Journal of Hypertension. 26(8):997-1004.

Falkner, B and Daniels, S. 2004. Summary of the Fourth Report on the Diagnosis, Evaluation, and Treatment of High BP in Children and Adolescents. American Journal of Hypertension. 44: 387-388.

Feig, D and Johnson, R. 2007. The Role of Uric Acid in Pediatric Hypertension. Journal of Renal Nutrition.17(1): 79-83.

Flynn, J and Tullus, K. 2009. Severe hypertension in children and adolescents: pathophysiology and treatment. Pediatric Nephrology. 24:1101-1112.

Ippisch, $\mathrm{H}$ and Daniels, S. Hypertension in overweight and obese children. 2008. Progress in Pediatric Cardiology. 25: 177-182.

Israeli, E; Schochat, T; Korzets, Z; Manova, D; Bernheim, J and Golan, E. Prehypertension and Obesity in Adolescents. American Journal of Hypertension. 2006; 19(7):708-712. 
Jones, ESW; Owen, P and Rayner, BL. 2012. The Association of the R563Q Genotype of the ENaC With Phenotypic Variation in Southern Africa. American Journal of Hypertension. 25(12): 1286-1291.

Jones, ESW and Rayner, BL. 2015. Hypertension, end-stage renal disease and mesangiocapillary glomerulonephritis in methamphetamine users. South African Medical Journal.105(3): 199-201.

Kagura, J; Adair, L; Musa, M; Pettifor, J and Norris, S. 2015. BP tracking in urban black South African children: birth to twenty cohort. BMC Pediatrics. 15(78):1-7. [Online]. Available: http://www.biomedcentral.com/1471-2431/15/78 [Accessed 18 July 2015].

Lim, S; Vos, T; Flaxman, A; Danaei, G; Shibuya, K; Adair-Rohani, H et al. 2012. A comparative risk assessment of burden of disease and injury attributable to 67 risk factors and risk factor clusters in 21 regions, 1990-2010: a systematic analysis for the Global Burden of Disease Study 2010. Lancet. 380(9859): 2224-2260.

Lu, Q; Ma, CM; Yin, FZ; Liu, BW; Lou, DH and Liu, XL. 2011. How to simplify the diagnostic criteria of hypertension in adolescents. Journal of Human Hypertension. 25:159-163.

Rayner, BL. 2010. Hypertension: Detection and Management in South Africa. Nephron Clinical Practice. 116: 269-c273.

Reddy, S; Resnicow, K; James, S; Funani, I; Kambaran, S; Omardien, RG; et al. 2012. Rapid Increases in Overweight and Obesity Among South African Adolescents: Comparison of Data From the South African National Youth Risk Behaviour Survey in 2002 and 2008. American Journal of Public Health. 102(2): 262268.

Seedat, YK; Rayner, BL \& Veriava, Y. 2014. South African hypertension practice guideline 2014. Cardiovascular Journal of Africa. 25(6): 288. 
Tirosh, A; Afek, A; Rudich, A; Percik, R; Gordon, B; Ayalon, N et al. 2010.

Progression of Normotensive Adolescents to Hypertensive Adults: A study of 26980 Teenagers. Journal of the American Heart Association. 56:203-209.

Tu, W; Eckert, G; DiMeglio, L; Yu, Z; Jung, J and Pratt, H. Intensified effect of Adiposity on BP in Overweight and Obese Children. American Journal of Hypertension. 2011; 58:818-824

Xi B, Zhang T, Zhang M, Liu F, Zong X, Zhao M, et al. 2015. Trends in Elevated BP Among US Children and Adolescents:1999-2012. American Journal of Hypertension. 2015:1-9.

World Health Organization. Global Status Report on Noncommunicable Diseases. 2014. 67-79. 